\title{
MARKETING DE INSTITUCIONES DEPORTIVAS: De las relaciones públicas a la valoración de la imagen corporativa como activo intangible*
}

\author{
Alfonso Gastañaduy Benel \\ PROFESOR DE MARKETING DE ESAN
}

\section{Resumen}

Este trabajo busca aportar al desarrollo de una mejor conceptualización de la gerencia de imagen corporativa aplicada a las empresas deportivas. El marco teórico presenta la evolución de los conceptos asociados a la imagen de la empresa, desde el surgimiento de las relaciones públicas. Luego se trata el caso específico de la gestión de grandes actividades deportivas, que incorpora los desarrollos modernos de gestión empresarial, fuertemente focalizados en el manejo de imagen y con clara vocación de aplicación de la teoría de la reputación; ello, a fin de lograr que el manejo financiero de los activos intangibles se materialice en una creciente y consistente valoración de la «marca» de la empresa deportiva o de la gran competencia deportiva internacional. Se describen experiencias reales de empresas exitosas en el campo del deporte.

\section{Marco conceptual}

1.1. Antecedentes: las clásicas relaciones públicas

En general, tratar acerca de las relaciones públicas es tratar sobre la forma y no sobre el fondo, porque las relaciones públi-

Ponencia presentada y expuesta por el autor en el Congreso Mundial de Gestión Económica del Deporte, Sport Congress 2003, organizado por la Escuela de Administración de Empresas de la Universitat Politécnica de Catalunya. Bar- cas aparecen cuando las empresas sienten la necesidad de influir sobre los públicos para lograr sus objetivos empresariales, en un estadio más avanzado de competencia. Antes de ello, en los inicios del sistema industrial moderno, cuando las necesidades estaban grandemente insatisfechas, los consumidores aceptaban con gusto lo que

celona, 14-16 de mayo de 2003. Publicada en los proceedings del congreso, puede verse en el sitio web siguiente:

http://www.sportcongress.org/Ponencias/ Gasta\%F1aduy. PDF. 
el sistema productivo les entregaba. No había exigencias, ni siquiera la necesidad de usar marcas. Los productos (bienes o servicios) eran lo que ahora se conoce como productos genéricos o indiferenciados. En aquellos tiempos, los gerentes de empresa tenían una gran preocupación, básica pero muy realista: ganar más por mayores ventas.

Más adelante, a mediados del siglo $\mathrm{XX}$, en plena postguerra e inicios del gran salto al consumo masivo en los Estados Unidos de América, los estudiosos del management comienzan a estructurar y generalizar todas las experiencias que se desarrollan por la ya intensa competencia empresarial. Se inicia la publicación masiva de libros de texto sobre la nueva ciencia de la empresa. Por ejemplo, Bethel y otros ${ }^{1}$ manifiestan que:

La gerencia industrial tiene por delante constantemente la tarea de comprender las condiciones y las relaciones entre los intereses privados y los de la sociedad. Una industria privada tiene que ser manejada en armonía con ambos intereses. La libertad personal, la seguridad de los derechos privados de la propiedad y la libertad de la iniciativa y de la empresa individuales, que son premisas esenciales de un sistema de empresa privado, dependen de una comprensión y de una adaptación adecuadas de los intereses de los individuos y los de la sociedad...

1. Bethel, Lawrence L.; Atwater, Franklin S.; Smith, George H. E.; Stackman, Harvey A., Jr. Organización y dirección industrial. Fondo de Cultura Económica, México. Primera edición en inglés: 1945; sexta edición en español: 1965. Pág. 19.
En esta afirmación puede observarse toda una sustentación del sistema de libre empresa, pero ya con una preocupación por los intereses de la sociedad. Y este aspecto, precisamente, sirve de punto de apoyo para el desarrollo de este trabajo: la responsabilidad de las gerencias de engranar los intereses de la empresa con los de la sociedad. A partir de este entendimiento se va tecnificando el manejo empresarial en muchos aspectos, lo que en aquellos tiempos da base más que a la importancia, a la necesidad de «quedar bien» con la sociedad. He ahí la simiente de las primeras concepciones sobre las relaciones públicas.

Luego de varias décadas, y también con más estructura en sus conceptos y principios, los investigadores de las relaciones públicas comienzan a ver más claro su panorama. Es el caso de Marston ${ }^{2}$ quien, para destacar la variedad de conceptos que existen sobre el tema, cita un artículo que apareció en Harper's Magazine y donde Robert Heilbroner se refirió a los especialistas de relaciones públicas como: «una hermandad de 100,000 integrantes cuyo lazo común es que ninguno de ellos estará jamás de acuerdo sobre lo que es su profesión». Pero el mismo Marston, luego de sesudas disquisiciones, plantea la siguiente definición:

Las relaciones públicas son la función gerencial que evalúa las aptitudes públicas, identifica las políticas y procedimientos de una organización con el interés público, y ejecuta un programa de acción [y comunicación] para

2. Marston, John E. Relaciones públicas modernas. México: McGraw-Hill. Primera edición en inglés: 1979; traducido al español: 1990. Págs. 5-6. 
ganar aceptación y entendimiento públicos.

Pero la pregunta es, ¿para ganar aceptación y entendimiento públicos solamente? Tanto Marston como la mayoría de autores coinciden en que se trata de generar en la sociedad simpatía y aceptación positiva por lo que hace la empresa. Prácticamente, ninguno considera que ello al final debe ser un valor intangible y hasta parte del patrimonio de la empresa.

\subsection{Los inicios: la promoción per se}

Paralelamente a estos desarrollos, por otro lado más funcional de la gestión empresarial, el comercial, se suscita una verdadera avalancha de autores y propuestas sobre lo que ahora se conoce como marketing. De sus antecedentes hay mucho que decir, pero es importante destacar que uno de los grandes aportes a esta ya madura disciplina es el de E. Jerome McCarthy, quien en su famoso libro Basic Marketing: A Managerial Approach ${ }^{3}$, publicado en 1960, hace conocer su famosa teoría de las 4 P: Product, Price, Place y Promotion, que se han difundido en español como Producto, Precio, Plaza y Promoción.

Más de tres décadas después, McCarthy ${ }^{4}$ mantiene su planteamiento inicial cuando afirma lo siguiente:

Las cuatro «P» constituyen una mezcla comercial. Un análisis de los pro-

3. McCarthy E., Jerome. Basic Marketing: A Managerial Approach. Homewood, IL: Richard D. Irwin, 1960

4. McCarthy E., Jerome y Perreault, William D. Comercialización; Basic Marketing. Buenos Aires: El Ateneo, 1992. Págs. 33-37. Versión en español de su texto primigenio de 1960 . blemas que enfrentan tanto las compañías grandes como las pequeñas demuestra la posibilidad de reducir el número de variables de la mezcla comercial a cuatro categorías básicas: Producto, Precio, Plaza y Promoción.

Más aún, ya en los inicios del tercer milenio, y a tono con los avances tecnológicos y de la era de la comunicación, McCarthy ${ }^{5}$ sigue reafirmando su indiscutido planteamiento:

Las necesidades de los consumidores meta pueden atenderse en muchas formas. Un producto puede reunir varias características. Es posible ajustar los niveles de servicio al cliente antes y después de la venta. Pueden cambiarse el empaque, el nombre de marca y la garantía. También es posible utilizar diversos medios publicitarios: periódicos, revistas, televisión por cable e internet. Puede usarse la fuerza de ventas de la compañía u otros especialistas. El precio está sujeto a modificaciones, descuentos, etcétera. Con tantas variables de donde elegir, ¿hay alguna forma de ayudar a organizar a todas estas decisiones y simplificar la selección de la mezcla de marketing? La respuesta es afirmativa. Conviene reducir todas las variables de la mezcla de marketing a cuatro fundamentales: Producto, Plaza, Promoción, Precio. Las cuatro Ps son un medio adecuado de concebir las cuatro partes de la mezcla de marketing.

Al respecto, y sin perder la perspectiva de este trabajo, es necesario hacer cuatro precisiones:

5. McCarthy E., Jerome y Perreault, William D. Marketing, un enfoque global. México: Irwin, McGraw-Hill, 2001. Pág. 47. 
- Con relación a Producto y Precio, no hay problema de traducción, pues ambos se refieren al satisfactor y a su valor de transacción.

- Sin embargo, Plaza no es lo que McCarthy quiso decir. Utilizó el término inglés Place, que significa lugar, ubicación, distribución. Plaza remite a «plaza mayor» o «plaza de armas» de una población o, simplemente, al mercado físico de toda ciudad («la plaza»). La confusión se originó porque los traductores, para mantener el acrónimo, tradujeron place como «plaza».

- En cuanto a Promoción, siempre en su citado texto de 2001, McCarthy reafirma su planteamiento de 1960 diciendo que:

... consiste en comunicar información entre el vendedor y el comprador potencial $\mathrm{u}$ otros miembros del canal para que influyan en las actitudes y en el comportamiento. El gerente de marketing se encarga de decir a los clientes que el producto idóneo está disponible en la plaza adecuada y al precio correcto... un ejecutivo de marketing puede seleccionar entre varios métodos promocionales: venta personal, venta masiva y promoción de ventas... debido a que cada método tiene sus virtudes y sus limitaciones... es necesario que los administre y coordine como un «todo» integrado, no como partes separadas e inconexas.

Ya en estos tiempos, pues, McCarthy habla de «comunicación integrada de marketing».

- La diferencia entre variables controlables y variables no controlables. El mismo McCarthy, ya en su citado libro de 1992, reafirma el sustento de su planteamiento, que muchos han olvidado o no han estudiado:

La planificación de la estrategia de un gerente comercial no se realiza en el vacío. Por el contrario, ese gerente opera con variables controlables, encuadradas en un marco que implica muchas variables incontrolables que se deben tener en cuenta, aunque el gerente no pueda controlarlas.

Las variables controlables son las ya mencionadas $4 \mathrm{P}$; las variables no controlables son los ambientes siguientes: Social, Económico, Político, Tecnológico y Ecológico (SEPTE).

Hechas las precisiones, y luego de una revisión de la más importante literatura que sobre marketing se ha publicado en las últimas décadas, y especialmente en los primeros años del tercer milenio, puede verse que las $4 \mathrm{P}$ de McCarthy mantienen actualísima vigencia: siempre habrá que vender un satisfactor, a un precio definido, en un lugar determinado y con la debida promoción. Sobre esta base, en la categoría Promoción, se observa que el enfoque es netamente comercial y con eficiencia probada.

Sin embargo, por otro lado ha habido un gran desarrollo de las ciencias de la comunicación, que han comenzado a perfeccionar los primeros alcances de la categoría Promoción. Un gran hito, poco conocido, es la revisión efectuada por Van Waterschoot \& Van den Bulte ${ }^{6}$, quienes

6. Van Waterschoot, Walter y Van den Bulte, Christophe. The 4P Classification of the Marketing Mix Revisited. Journal of Marketing: A Quarterly Publication of the American Marketing Association. October 1992, vol. 56, n. ${ }^{\circ} 4$, págs. 83-93. 
plantean la siguiente visión mejorada de las $4 \mathrm{P}$ o marketing mix: Producto, Precio, Distribución y Comunicación. En la variable Comunicación hacen el desagregado siguiente: comunicación masiva, comunicación personal y publicity. A la venta personal, la denominan comunicación personal, y añaden el publicity o comunicación no pagada. Plantean que la clásica categoría Promoción está contenida en toda la mezcla comercial o marketing mix. Ello, porque la Promoción per se, que con el tiempo fue viéndose y aplicándose como un instrumento muy concreto, es incorporada por Van Waterschoot \& Van den Bulte en todas las categorías componentes de las $4 \mathrm{P}$; de ahí que propongan que hay: mix de promoción de producto, mix de promoción de precio, mix de promoción de distribución, mix de promoción de comunicación masiva, mix de promoción personal y mix de publicity.

\subsection{Los primeros avances: las decisio- nes estratégicas de comunicación}

En este ampliar del alcance de la categoría Promoción, por el desarrollo de las ciencias de la comunicación y por el del posicionamiento, gran aporte es el de Ries $\&$ Trout, ${ }^{7}$ quienes dicen que:

... el posicionamiento comienza con un producto, que puede ser un artículo, un servicio, una compañía, una institución o incluso una persona [...] es también lo primero que viene a la mente cuando se trata de resolver el problema de cómo lograr ser escuchado en

7. Ries, Al y Trout, Jack. Posicionamiento: el concepto que ha revolucionado la comunicación publicitaria y la mercadotecnia. México: McGraw-Hill de México, 1985. Traducción de la primera edición en inglés de Positioning: The Battle for Your Mind. McGraw-Hill Book, 1981. una sociedad supercomunicada [...] En comunicación, lo menos es más...

Puede apreciarse, pues, que ya se prueba científicamente que la necesidad de información del mercado o conjunto de consumidores se satisface con un gran compuesto decisional que se llama comunicación empresarial, y que éste abarca mucho más allá del mero bien o servicio comercial.

En esta línea, Jean-Jaques Lambin ${ }^{8}$, uno de los primeros especialistas del marketing estratégico llama Comunicación a esta categoría, cuya finalidad la resume en dos grandes objetivos por lograr en el mercado: hacer conocer y hacer valer la oferta de la empresa. Define a la comunicación como:

... el conjunto de señales emitidas por la empresa a sus diferentes públicos. Es decir, hacia clientes, distribuidores, proveedores, accionistas, poderes públicos y también frente a su propio personal.

En este sentido, los medios de comunicación en marketing son: publicidad, fuerza de ventas, promoción de ventas y las relaciones exteriores. En cuanto a las relaciones exteriores, Lambin afirma que:

... tienen por objetivo establecer, a través de un esfuerzo deliberado, planificado y sostenido, un clima psicológico de comprensión y de confianza mutua entre una organización y el pú-

8. Lambin, Jean-Jaques. Marketing estratégico. Madrid: McGraw-Hill/Interamericana de España, 1995. Tercera edición en español. Traducción de la tercera edición en francés de Le Marketing Strategique. París: Ediscience International, 1994. Págs. 519-568. 
blico. Se trata pues menos de vender que de obtener un apoyo moral que facilite la continuidad de la actividad.

Se acerca Lambin al tema de este trabajo al desarrollar todo lo que significa el proceso de la comunicación publicitaria. Señala que los diversos tipos de comunicación publicitaria son: publicidad de imagen, publicidad promocional, publicidad interactiva y publicidad institucional. Destaca que los tres primeros tipos de comunicación publicitaria precedentemente indicados están referidos al satisfactor que la empresa ofrece al mercado, y que la publicidad institucional:

... tiene por objetivo crearse o reforzar una actitud positiva hacia la empresa, dirigiéndose a sus diferentes públicos [...] se trata, pues, también de un objetivo de creación de imagen, pero de una imagen de empresa.

A partir de esta afirmación, Lambin indica que la publicidad institucional tiene dos principales modalidades particulares: el patrocinio (operación comercial de mutuo beneficio entre las partes) y el mecenazgo (donde prima el carácter desinteresado y generoso). A estas alturas, las ciencias de la comunicación se han incorporado al marketing y lo han enriquecido, haciendo ver que no sólo debe comunicarse el satisfactor, sino también una buena imagen de la empresa en su totalidad.

Reafirma lo dicho el siempre renovado Philip Kotler, cuando en su libro $\mathrm{Di}$ rección de marketing, edición del milenio ${ }^{9}$ denomina la categoría como Comunica-

9. Kotler, Philip; Cámara, Dionisio; Grande, Ildefonso; Cruz, Ignacio. Dirección de Marketing. Edición del Milenio. Madrid: Pearson Educa- ción integral de marketing, cuya definición adopta de la Asociación Americana de Agencias de Publicidad:

Un concepto de planificación de comunicaciones en marketing que reconoce el valor añadido de un plan completo que evalúa los roles estratégicos de una variedad de disciplinas de comunicación [...] y que combina estas disciplinas para proporcionar claridad, consistencia y el máximo impacto de las comunicaciones a través de la integración uniforme de mensajes discretos.

Señala Kotler que esta categoría cuenta con cinco herramientas principales: publicidad, promoción de ventas, relaciones públicas, venta personal y marketing directo. Si bien es cierto que habría un aparente retroceso conceptual en Kotler al reconocer a las relaciones públicas como herramienta principal de comunicación integral de marketing, no lo es tal por la aclaración que hace:

Los directores de marketing y los especialistas en relaciones públicas no siempre hablan el mismo lenguaje. Los primeros van más al grano, mientras que los segundos contemplan su trabajo como comunicadores, si bien la situación está cambiando: en primer lugar, las empresas están pidiendo más relaciones públicas en marketing (RPM), prestando apoyo directo a la promoción de la empresa o del producto y a la creación de imagen. Así, las RPM, lo mismo que las relaciones públicas en los aspectos financieros y

ción, 2000. Primera edición en español, traducción de Marketing Management. Prentice Hall, 2000 
en otros departamentos, podrían servir para lograr objetivos concretos, en este caso el desarrollo del departamento de marketing... El nombre primitivo de las relaciones públicas en marketing era 'publicidad', a la que se contemplaba como la tarea de conseguir espacio gratuito en la prensa y en la radio con objeto de promocionar o dar imagen de un producto, servicio, idea, lugar, persona o empresa...

Y reafirma Kotler que las RPM, entre otras netamente comerciales, pueden contribuir a tareas como: influenciar a grupos objetivo específicos (patrocinios), defender productos que se han enfrentado a problemas públicos (intoxicación por Coca Cola en Bélgica) y crear imagen corporativa de un modo tal que afecte favorablemente a sus productos (IacoccaChrysler).

Estamos, pues, de lleno en el manejo o gerencia de la imagen corporativa, de la que se comienza a observar un torrente de publicaciones, la mayoría casi totalmente orientada a los aspectos exclusivamente técnicos (comunicacionales) y con clarísimo sesgo operativo y no de rentabilidad.

\subsection{EI desarrollo de las ciencias de la comunicación: gerencia de la imagen corporativa}

En el mundo empresarial, ya es una práctica común hablar de gerencia y gerente de Imagen Corporativa, en lugar de «jefe de Relaciones Públicas». Pero también se puede observar que en la mayoría de los casos se trata de un simple cambio de nombre, algo equivalente a ejecutivo de negocios en lugar de vendedor. Una revisión rápida de los principales aportes bibliográficos sobre el tema permite afirmar que se ha avanzado algo en cuanto a la importancia del manejo de la imagen de la empresa, pero en sus aspectos operativos o de procedimientos.

Sin embargo, también se encuentran aportes empresariales; es decir, en función del objetivo final del hacer empresa: maximizar la rentabilidad de la inversión o, lo que es lo mismo, incrementar el valor real de sus acciones comunes. Un ejemplo de ello es lo que afirma Justo Villafañe ${ }^{10}$ :

... de nada sirve analizar [...] la imagen que de una compañía proyectan los medios de comunicación -que se corresponderá con su perfil mediático exclusivamente- si no se integran en el mismo modelo de análisis otros aspectos igualmente importantes para su imagen, como puede ser su reputación financiera, el valor de sus marcas, el trato que sus empleados de contacto dispensan a sus clientes o la misma cohesión interna de su plantilla, por citar sólo algunas variables que influyen en la imagen de una empresa.

Si esto ya se está entendiendo en las empresas, en buena hora, porque lo implícito comienza a ser explícito en este quehacer de manejar la imagen corporativa: el enfoque empresarial o, específicamente hablando, el enfoque financiero del marketing en su variable comunicacional. Esto significa que la gerencia de la imagen corporativa no es sino el incrementar la reputación empresarial, la misma que se puede valorizar pues, de hecho, ya es parte del patrimonio de la firma.

10. Justo Villafañe. La gestión profesional de la imagen corporativa. Madrid: Pirámide, 1999. Pág. 46. 


\subsection{De la imagen corporativa a la reputación empresarial: valor patrimonial de la reputación}

Al respecto, Fombrun ${ }^{11}$ afirma que la reputación es valiosa porque nos informa sobre qué productos comprar, qué compañías trabajar o en qué acciones invertir. Al mismo tiempo -sigue Fombrun-, una reputación es de considerable valor estratégico porque llama la atención sobre los rasgos atractivos de una compañía y amplía las opciones disponibles a sus gerentes, sea para fijar precios más altos o más bajos a los productos y servicios o para implementar programas innovadores.

La buena imagen corporativa significa buena reputación corporativa. Buena reputación corporativa significa mayor valor patrimonial. Y este mayor valor patrimonial es la resultante de las interrelaciones entre la identidad de una empresa y su nombre, imagen y reputación. A su vez, la imagen corporativa se manifiesta en varios planos: imagen en el cliente, imagen en la comunidad, imagen en los accionistas, imagen en el personal. Éstos son los razonamientos de Fombrun para afirmar que la reputación debe construirse, sostenerse y defenderse; es decir, debe gerenciarse. Redondea su razonamiento cuando conceptúa sobre los activos intangibles (goodwill), los mismos que, por los principios contables de los Estados Unidos, no se pueden valorizar como activos, lo que ha permitido que los contadores hayan acordado reconocer el goodwill sólo cuando la firma es vendida. Ellos miden el valor de los activos intangibles como el exceso del precio de compra de la com-

11. Fombrun, Charles J. Reputation: Realizing Value from the Corporate Image. Boston, Massachusetts: Harvard Business School Press, 1996. pañía sobre el valor de mercado de sus activos tangibles. El goodwill típicamente incorpora el valor de todos los intangibles, incluyendo nombres de marca y reputación.

Pero Hans Frank ${ }^{12}$ puntualiza que los contadores, con un criterio conservador, prefieren:

... contabilizar operaciones relacionadas con intangibles directamente como gastos del periodo, antes que mantenerlas como activos intangibles, amortizándolos en periodos de mayor amplitud.

De otro lado, los contadores generalmente no toman en cuenta los activos intangibles por razones de índole tributaria, pues prefieren contabilizarlos como gastos del periodo antes que activarlos considerándolos como una inversión.

Pero más allá de la visión contable, se debe apreciar la visión empresarial, que es desde donde se evidencia el concepto de capital intelectual.

\subsection{El capital intelectual}

Avanzando o profundizando en el concepto de activos intangibles, se llega rápidamente a otro más amplio y crecientemente más conocido: el capital intelectual de las empresas. Stewart ${ }^{13}$ lo define de la siguiente manera:

12. Frank, Hans. Los activos intangibles: control y contabilización. Lectura complementaria preparada para el curso de Contabilidad Gerencial en la Escuela de Administración de Negocios para Graduados (ESAN), Lima, 2001.

13. Stewart, Thomas A. La nueva riqueza de las organizaciones: el capital intelectual. Barcelona: Granica, 1998. Pág. 10. 
El capital intelectual es material intelectual -conocimientos, información, propiedad intelectual, experiencia- que se puede aprovechar para crear riqueza. Es fuerza cerebral colectiva. Es difícil de identificar y aun más de distribuir eficazmente. Pero quien lo encuentra y lo explota, triunfa.

Dice este estudioso que ello es fácil de entender por la simple observación del «contenido intelectual» de bienes y servicios cotidianos, ya que en todo hay contenido intelectual: un envase con identidad (marca) no es un simple envase físico, un viaje en Iberia no es un simple traslado aéreo de un lugar a otro en una línea desconocida, un clásico Barcelona-Real Madrid no es un simple partido de fútbol más..., las casaquillas del Barça o del Real Madrid no son simples prendas de vestir: significan mucho más, valen mucho más para el público, para los clubes..., para la Bolsa.

Pero la conceptualización de Stewart no puede adoptarse a secas. Ello, porque Edvinson y Malone ${ }^{14}$ han estudiado el tema y encontrado las ideas siguientes:

El capital intelectual no solamente incluye el potencial del cerebro humano, sino también los nombres de productos y las marcas de fábrica y hasta gastos registrados en los libros como históricos, que se han transformado con el correr del tiempo en algo de más valor (por ejemplo, un bosque comprado hace un siglo, que hoy es una va-

14. Edvinson, Leif y Malone, Michael. El capital intelectual: cómo identificar y calcular el valor de los recursos intangibles de su empresa. Barcelona: Gestión 2000, 1999. Págs. 17-19. liosísima propiedad inmobiliaria). Son activos que en la actualidad están valorados en cero en el balance general. Steven M.H. Wallman, presidente de la Comisión de Valores y Bolsa.

Tenemos necesidad de pasar a un nuevo nivel contable, en el cual se pueda medir el impulso de una empresa en términos de posición de mercado, lealtad de la clientela, calidad, etc. Por no valorar estas perspectivas dinámicas estamos una valoración tan falsa de una empresa como si estuviéramos cometiendo errores de suma. William Davidow, inversor de capitales para especulación y comentarista de negocios (The Virtual Corporation).

... el capital intelectual se esconde dentro de ese concepto contable tradicional y misterioso llamado goodwill. La diferencia es que tradionalmente el goodwill recalcaba activos poco usuales pero reales, tales como las marcas de fábrica. En comparación, el capital intelectual busca activos todavía menos tangibles, tales como la capacidad de una empresa para aprender y adaptarse. H. Thomas Jonson, profesor de Administración de Empresas en la Universidad de Portland (Oregon).

Los directivos se esfuerzan aquí y ahora por adaptarse al desplazamiento del centro de gravedad económico, de administrar y medir los activos materiales y financieros, a cultivar y multiplicar el conocimiento como los actos más significativos de creación de valor. Y esto se aplica tanto a Microsoft como a una fábrica de tejidos en los desiertos de Canadá que teje boinas en una máquina comprada por el bisabuelo del propietario en 1919. 
Pregúntele a un ejecutivo de cualquier firma qué porcentaje del valor total atribuiría a los activos intangibles -cualquier cosa, desde destrezas individuales y conocimientos técnicos, sistemas de información, diseños y marcas de fábrica, hasta relaciones con los proveedores y concesionarios- y recibirá la misma respuesta: más del $80 \%$. Ahora pida que observe la relación entre el fondo de comercio y los activos realmente incluidos en el balance general, y le producirá un ataque nervioso. Revista Industry Week, a principios de 1996.

Toda nuestra contabilidad, todos los reglamentos del gobierno y de la bolsa de valores, todos los recursos, todo está enfocado en el capital social, lo que es absolutamente absurdo porque los 2,000 millones de coronas danesas de capital intelectual son cinco veces más. La revista Industry Week, citando a Lars Kolind, presidente de Oticon Holding A/S, firma danesa fabricante de audífonos que ha crecido en valor de mercado de 150 millones de coronas en 1991 a 2,400 hoy (1997), y sin embargo sólo 400 millones de esa suma aparecen en el balance general.

Con estas referencias, Edvinson y Malone afirman que:

Cualquiera que sea la definición que se adopte, es evidente que el valor del capital intelectual en los negocios mundiales es inmenso. Charles Handy calcula que puede llegar a tres o cuatro veces el valor de los activos de una empresa. De acuerdo con el Indice Mundial Morgan Stanley, el promedio del valor de las empresas en las bolsas de valores mundiales es el doble de su valor en libros. En Estados Unidos el valor de mercado de una corporación es normalmente de dos a nueve veces su valor en libros.

Cuando citan en su libro la fórmula del capital intelectual de James Tobin, estos especialistas coinciden con la referencia que Fonbrum hace de los contadores en Estados unidos:

$$
\mathrm{CI}=\mathrm{VM}-\mathrm{VL}
$$

Donde:

CI: capital intelectual.

VM: valor de mercado.

VL: $\quad$ valor en libros.

\subsection{Los desarrollos y retos financieros: la valoración del capital intelectual}

De todo lo revisado hasta este punto, puede concluirse que el primigenio concepto de goodwill referido a los activos intangibles obvios, explícitos o reales, ha dado paso al concepto más amplio de capital intelectual en las empresas.

Ello lleva a plantear que el gran reto en estos tiempos es cómo valorar dicho capital. Hay muchas metodologías y criterios. Pero la pregunta es: ¿cuál es el punto en el que debe fijarse o entenderse una fórmula o metodología? Al respecto, ayuda muchísimo la afirmación de Vega, Bonilla y Cebrián ${ }^{15}$ :

15. Vega, Monserrat; Bonilla, María Jesús; Cebrián, Mónica Santos. Algunas reflexiones sobre la contabilidad de costes para activos intangibles. Harvard Deusto, Finanzas \& Contabilidad. 2001, nov.-dic., n. ${ }^{\circ} 44$. 
... existe una tendencia generalizada a considerar que los intangibles deben ser calificados como activos por su capacidad potencial para generar beneficios futuros y, por tanto, con proyección plurianual... Desde un punto de vista económico e independientemente de que en un futuro más o menos próximo los intangibles figuren activados en los estados contables, su valoración a coste de producción entrañaría... los problemas siguientes: a) reconocer el intangible como fuente de la ventaja competitiva que disfruta su compañía..., b) identificar los insumos asociados a los activos intangibles..., c) ¿cuándo acaba el proceso de acumulación de costes?..., d) (...) habría que identificar el período durante el cual la empresa se beneficia del uso del intangible.

Estos razonamientos hacen ver que el tema es cuasi filosófico. Los especialistas se pondrán de acuerdo en su momento. Para los objetivos de este trabajo, al final de cuentas el valor se definirá en el momento de realización de todos los activos de la empresa (físicos y capital intelectual): cuando se venda la empresa, cuando se efectúe una transacción documentada. En ese momento se encajará con las Normas Internacionales de Contabilidad (NIC) o con los Principios de Contabilidad Generalmente Aceptados (PCGA). A propósito, la Unión Europea ha incorporado recientemente las NIC, pero el país más poderoso y con filiales en casi todo el mundo, Estados Unidos, sólo acepta los PCGA.

Pero, siempre con la visión de marketing como filosofía de hacer negocios, es importante destacar que el valor más importante, el determinante, es el valor del mercado, entendido éste como el valor que los consumidores finales o usuarios industriales dan a una empresa al comprar sus productos o servicios: su imagen, su reputación, sus marcas, sus sistemas de distribución, su calidad de servicio, su solvencia, su cumplimiento, etc.; es decir, el encanto de los «momentos de la verdad» (momento de interacción real entre el cliente y el prestador del servicio) al recibir soluciones o satisfacciones esperadas, o más que eso, y que les hace ser fieles consumidores a través del tiempo. Entonces, en esto es muy importante también el concepto de valor del consumidor en el tiempo, como generador de ventas o aportes a la rentabilidad de la empresa y, por ende, al mayor valor patrimonial de ésta. Nada valdrán los componentes del capital intelectual si el consumidor no los valora a través del tiempo.

\section{Implicancias de la valoración de activos intangibles en la gestión de instituciones deportivas}

\subsection{Casos de sistemas empresariales en el deporte}

¿Cómo valorar el encanto de una gran empresa como los juegos olímpicos? ¿Cómo valorar el encanto de participar en un campeonato mundial en cualquier disciplina deportiva? ¿Cómo valorar lo que significa la camiseta del Barça, del Real Madrid, del Manchester United, por citar algunos brillantes ejemplos?

Con esta ponencia se trata de demostrar que el valor de una institución o certamen deportivo es más que sus inventarios físicos o lo que registran sus libros contables. Desde el punto de vista de la gestión económica, el valor de una insti- 
tución deportiva estará determinado por los componentes de su propio capital intelectual: conocimientos, información, propiedad intelectual, experiencia. Pero, más propiamente, el valor de una institución deportiva estará dado por muchos otros factores, entre los que se pueden destacar los siguientes:

- Su rendimiento o performance en competiciones y, obviamente su posición destacada a través del tiempo.

- Su gestión del merchandising, con gerencia profesional y con un gran enfoque de marketing estratégico; es decir, en función del mercado.

- Su infraestructura, con los modernos conceptos de estadio-empresa desarrollados especialmente por poderosas instituciones deportivas en los Estados Unidos.

- La calidad y prestigio de su plantel de jugadores, que, con buen manejo de su imagen profesional y personal, contribuye a valorar la institución de manera inconmensurable.

- Sus patrocinadores, que mientras más poderosos sean potenciarán la imagen corporativa y, por ende, el valor patrimonial de la institución deportiva. Ello debido a que un patrocinador de marca internacional no invertirá en una institución deportiva que no sea también poderosa.

- Su plantel gerencial, pues sin una solvente y profesional conducción empresarial ninguno de los aspectos indicados funcionará.
Todo lo desarrollado en esta ponencia es el reflejo de realidades empresariales de mucho éxito en la gestión económica del deporte. A manera de ejemplo, se pueden mencionar las experiencias siguientes:

a) El éxito de la estrategia The Olympic Partnership, de Joan Antonio Samaranch, ex presidente del Comité Olímpico Internacional (COI) ${ }^{16}$

- El COI ha sido la única transnacional deportiva que no ha sufrido los estragos del enfriamiento de la economía, acentuado en los últimos días por los actos terroristas contra los intereses norteamericanos. El secreto: Joan A. Samaranch estructuró una sólida base financiera de largo plazo a la que bautizó con el nombre de programa TOP (The Olympic Partnership), con socios corporativos solventes, principalmente multinacionales.

- Según las cifras hechas públicas por la revista Marketing Matters, publicada por el COI, la organización deportiva solamente pudo entregar en 1980, 88 millones de dólares a los comités de organización de los Juegos de Moscú (verano) y de Lake Placid (invierno). Pero en el 2000, el plan Samaranch distribuyó a los organizadores de Sydney (verano) 1100 millones de dólares, y 575 millones de dólares a los de Salt Lake City (invierno 2002). Una progresión de $1450 \%$.

16. Caballero, Ramón. El éxito de la estrategia The Olympic Partnertship. Madrid, 12 de nov., 2001. Publicado en la revista electrónica DEPORTE\&NEGOCIOS ${ }^{\mathrm{TM}}$, Diario Económico y Financiero del Deporte. Autorizada su cita al autor de este trabajo por DEPORTE\&NEGOCIOS, (C) D\&N (contactenos@deporteynegocios.com). 
- El apoyo financiero del que se benefician las federaciones internacionales, que perciben un porcentaje sobre los derechos de retransmisión televisada, pasó de 8 millones de dólares (juegos de invierno y de verano de 1980) a 255 millones (juegos de invierno de 1988 y de verano de 2000).

- En total, el COI redistribuye ahora el 93\% de sus ingresos a los comités de organización, a los comités nacionales olímpicos (CNO) y a las federaciones.

- Los ingresos proceden sobre todo de los derechos de televisión. En los Juegos de verano de Moscú de 1980, se elevaban a 101 millones de dólares. En Sydney, en el 2000, alcanzaron los 1300 millones de dólares.Y rozarán los 1500 millones de dólares en los de Atenas-2004, mientras que los contratos firmados para el 2008 prometen 1700 millones de dólares. Con esta apertura comercial, los Juegos Olímpicos se han convertido en un apetecido socio corporativo de multinacionales que observan en la popularidad de la fiesta deportiva una ocasión para multiplicar la imagen de sus marcas y productos.

b) La estrategia norteamericana estadio-empresa, foco de desarrollo comercial ${ }^{17}$

- El deporte norteamericano agrega a su atractivo portafolio otro producto rentable: el estadio-empresa. Obras gigantes con una amplia oferta de «ganchos

17. Martínez, Hugo H. La estrategia estadio-empresa, foco de desarrollo comercial. Miami, 3 de ene., 2003. Publicado en la revista electrónica DEPORTE\&NEGOCIOS ${ }^{\mathrm{TM}}$, Diario promocionales» de los equipos y patrocinadores para cautivar el interés de los consumidores. Son centros comerciales dotados de servicios bancarios, hoteleros, restaurantes, salas de conferencias y apuestas, suites y tiendas, entre algunas de las muchas ofertas.

- El negocio consiste en alianzas estratégicas que los gobiernos locales y las franquicias de las ligas profesionales ponen en marcha con gran impacto en las economías de los condados por la generación de fuentes de empleo, ingresos fiscales y fomento de la recreación; además de los rendimientos económicos y logísticos propios del ejercicio empresarial.

- En el marco de este nuevo orden comercial, en 1999 los San Antonio Spurs, franquicia de la NBA, y el gobierno de esta ciudad texana fusionaron esfuerzos para construir el SBC, estadio-empresa ubicado a más de 10 millas del centro del Riverwalk, con 18500 sillas y 56 suites. La financiación inicial fue asumida por los habitantes de la ciudad con la aceptación de un impuesto sobre los servicios hoteleros y el alquiler de vehículos, aval con el cual el gobierno local trasladó recursos en monto de 146,5 millones de dólares; el equipo aportó $175 \mathrm{mi}$ llones de dólares. El retorno de la inversión comenzó a finales del 2002, cuando la obra se transformó en un gran empleador, efectivo contribuyente del fisco y escenario de los espectáculos.

Económico y Financiero del Deporte. Autorizada su cita al autor de este trabajo por DEPORTE\&NEGOCIOS, (C) D\&N (contactenos@deporteynegocios.com). 
- Otro escenario del nuevo negocio es el de los Dallas Stars de la NHL y los Dallas Mavericks de la NBA, que comparten el American Airlines Center. Pagan 6,5 millones de dólares de arriendo al año mediante un contrato extendido hasta el 2031, acuerdo de largo plazo que les permite la venta de sus 142 lujosas suites por temporada a 150 mil y 300 mil dólares cada una, y las sillas preferenciales a 7 mil y 18 mil dólares. La seriedad de la alianza entre la ciudad y los equipos motivó que American Airlines tomara el nombre del estadio y que los derechos de televisión de los dos equipos estén garantizados durante los próximos diez años por 195 millones de dólares.

- Otro caso productivo de esta experiencia comercial es la de los Sacramento Kings y las Sacramento Monarchs con el Arco Arena. En 1988, con una financiación privada de 40 millones de dólares, el Arco Arena aseguró la construcción de restaurantes y suites de lujo. Hoy, el estadio-empresa admite espectáculos circenses, conciertos, shows sobre hielo, competencias de motocross y juegos de playoff del baloncesto colegial. En el 2001, con la realización de 12 conciertos, los equipos captaron 12 millones de dólares, indicador que movió al departamento de mercadeo a realizar, durante el 2002, unos 170 espectáculos distintos a los juegos de las ligas profesionales de baloncesto (femenina y masculina). Sacramento Kings y Monarchs pagan 750 mil dólares de arriendo al año. El Arco Arena dispone de 30 suites de lujo cuyo valor individual por temporada es de 200 mil dólares. c) El modelo empresarial de la $N B A^{18}$

- Mientras la National Basketball Association (NBA) goza de espléndida salud financiera y un reconocimiento de marca que trasciende en más de 180 países, otro producto de la industria del deporte norteamericano atraviesa su peor crisis económica, a punto de un conflicto laboral de impredecibles consecuencias: la Liga Profesional de Béisbol (MLB), holding que administra 32 franquicias del béisbol profesional.

Empresarios y jugadores no han llegado a un acuerdo sobre el discutido tope salarial y el llamado «impuesto de lujo» que se quiere cobrar a aquellos equipos que sobrepasen los 98 millones de dólares de nómina, situación que acentúa el drama financiero de 450 millones de dólares que la MLB carga en pasivos.

- En cambio la NBA sonríe. Los ingresos combinados por temporada llegan a 5 mil millones de dólares, provenientes de innumerables artículos auxiliares y de sus conexiones promocionales, derechos de televisión y entradas, una rica fuente alimentada por acuerdos de licencias y patrocinios con más de 150 compañías, entre las que se cuentan gigantes como Coca-Cola, McDonald's y Nike; al tiempo que los coliseos se llenan al 92\%, abre ofici-

18. Luckas Emilio Azcárraga. Las diferencias de gestión de los modelos empresariales NBA y MLB. Nueva York, 15 de ago., 2002. Publicado en la revista electrónica DEPORTE-\&NEGOCIOS $^{\mathrm{TM}}$, Diario Económico y Financiero del Deporte. Autorizada su cita al autor de este trabajo por DEPORT\&NEGOCIOS, C D\&N (contactenos@deporteynegocios.com). 
nas en ocho ciudades fuera de los Estados Unidos, y sus juegos forman parte del consumo televisivo en cinco continentes.

- Si bien la liga constituye una confederación de 29 franquicias independientes, cada una con su propia organización de mercadeo, la NBA les brinda una protección unificadora que todavía no se ve en otros deportes profesionales. Hace algunos años, por ejemplo, cuando Minnesota Timberwolves tuvo dificultades financieras y se propuso un cambio de franquicia, la NBA envió a docenas de gerentes senior a Minneapolis, a fin de que diseñaran la venta de la franquicia a inversores locales y, de esa manera, el equipo siguiera en el mismo lugar. A cambio del apoyo que reciben, los equipos se mantienen fieles a la filosofía de mercadeo y de marcas de la liga. Saben que se trata de alimentar una máquina que genera ganancias para todos.

- Para cimentar su sólida estructura empresarial, la NBA tomó el modelo de mercadeo de Disney y McDonald's, y construyó un conglomerado de entretenimiento que extendió la marca a docenas de productos licenciados, tal como lo hace Disney con los fabricantes de juguetes y ropa. Entonces, la NBA se ubicó en el mercado como una marca, en lugar de concebirse como una asociación de clubes. Además, aunque los atletas profesionales habían sido considerados héroes y modelos de liderazgo, en un esfuerzo claro e intencional para elevar a las estrellas más allá de ese nivel, y convertirlas en celebridades, encontró en la televisión el socio ideal.
- Mientras el comisionado de la NBA, David Stern, es exaltado como el gran «gurú» que transformó un conjunto de franquicias débiles en una máquina de hacer dinero finamente aceitada, que ejercita sus músculos dentro y fuera de la cancha, su colega Bud Selig, comisionado de la MLB, no encuentra salida inmediata para solucionar los varios problemas económicos y legales que golpean a la industria del béisbol de las grandes ligas.

\subsection{Los clubes como empresas deportivas}

En el caso concreto de las instituciones o clubes deportivos, hay también experiencias de mucho éxito que es necesario estudiar más a fondo. A manera de ejemplo, se pueden mencionar las siguientes:

a) Fútbol Club Barcelona

Planes de acción previstos para el $2003^{19}$

Estos planes tienen como objetivo la generación de nuevos ingresos, la potenciación del área deportiva, la modernización de la gestión y la mejora de las prestaciones que se ofrecen a los socios y simpatizantes de la entidad.

Las medidas son tanto de gran alcance como de dimensiones reducidas, si bien todas son necesarias para la modernización y contribución a la expansión global de la entidad y con su difusión se pretende que los barceloneses tengan pleno conocimiento de todas las iniciativas de la gestión del Club.

19. Francisco Ramos Alcoba, Director Gestionydeporte.com framos@gestionydeporte.com. El FC Barcelona trabaja en la ejecución del conjunto de Planes de Acción previstos para el 2003. 
- Planes orientados al incremento de ingresos:

- Comercialización de los productos de la marca 'Barça' en 141 países.

- Explotación comercial de las instalaciones.

- Desarrollo de cinco líneas de negocio a través de la web.

- Incorporación de nuevos contenidos e iniciativas comerciales en el Museo.

- Comercialización de un amplio paquete de servicios a través de la telefonía móvil.

- Potenciación del servicio 'Seient Lliure'

- Ofensiva para incrementar el valor de la marca 'Barça'.

- Ampliación de los canales de distribución de Gent del Barça.

- Los productos 'Barça' llegarán al Japón por primera vez en la historia.

- Creación de nuevas ofertas de ocio.

- Potenciación de las giras tanto en España como en el extranjero.

- Ampliación de la oferta de restauración.

- Redefinición de los palcos del Camp Nou.

- Planes orientados a impulsar el área deportiva:

- Inversión de 9 millones de euros en el fútbol base y en la formación de jugadores.

- Nueva política de contratación de jugadores.

- Construcción de la Ciudad Deportiva «Joan Gamper».

- Reforzamiento de la cobertura médico asistencial a los deportistas.

- Planes orientados a mejorar la eficiencia de la gestión:
- Aplicación de un ambicioso plan tecnológico.

- Control de los gastos ordinarios no deportivos.

- Implantación de una cultura corporativa.

- Reforzamiento de las medidas de seguridad.

- Obtención de la certificación de calidad ISO 9001.

- Planes orientados a incrementar las prestaciones sociales:

- Ampliación de las facilidades para los desplazamientos de los aficionados con el equipo.

- Incremento de las actividades dirigidas al público infantil.

- Aumento de la información y servicios a través del correo corporativo.

- Nuevas inversiones de la Fundación por valor de 7 millones de euros.

Estrategia para incrementar el valor de la marca Barça ${ }^{20}$

- EL Fútbol Club Barcelona, a través de la sociedad FCB Merchandising SL (FCBM), creada a raíz de los acuerdos con Nike, ha puesto en marcha una ofensiva para incrementar el valor de la marca Barça por todo el mundo.

- La estrategia se desarrollará este año 2003 en cuatro frentes:

- Impulso del número y la calidad de las licencias oficiales.

- Expansión de la distribución local e internacional.

20. El Barça impulsa una ofensiva para incrementar el valor de su marca. Artículo publicado en el sitio web http://www.gestionydeporte.com/ noticia66.htm. 
- Consolidación de la venta a través de Internet.

- Remodelación de las instalaciones comerciales (La Botiga).

- FCBM cuenta con un equipo profesional especializado en el sector que, desde sus oficinas en el mismo Camp Nou, y junto a la dirección comercial y de marketing del Club, se responsabiliza de toda la expansión del negocio.

- Impulso a las licencias oficiales

La estrategia comercial del FCBM busca beneficiar a sus licenciatarios, dado que el negocio de licencias oficiales del Barça está todavía lejos de su potencial real. Esta estrategia se basa en cinco objetivos prioritarios:

- Reforzar, ampliar y mejorar la distribución de los productos oficiales.

- Establecer la línea de diseño con base en la nueva imagen corporativa del Club.

- Definir un estándar de calidad para los productos oficiales.

- Incluir nuevas categorías de productos, que a la vez sean atractivas para el consumidor.

- Aprovechar las sinergias de expansión internacional con el Manchester United y, a partir de la próxima temporada, con la Juventus de Turín.

- Expansión internacional de los productos FCB

Para el 2003 serán prioritarios tres mercados específicos: Europa, Asia y Estados Unidos.

- Mercado europeo: potenciar la distribución de productos, sobre la base de la sólida infraestructura comercial de Nike en toda Europa, y aprovechando la notoriedad y prestigio del Club en el continente.

- Mercado asiático: la estrategia se basa en determinar de manera selectiva en qué países se trabaja y con qué socios. Se prevé un acuerdo con un licenciatario único del Japón, que supondrá una fuente de ingresos adicionales a través de la venta de productos del merchandising oficial del Club.

- Mercado de los Estados Unidos: se obtendrán los primeros frutos durante la próxima pretemporada, cuando el primer equipo de fútbol realice una gira para enfrentarse entre otros, al Manchester y a la Juve. En este aspecto, FCBM establecerá acuerdos con empresas de distribución norteamericanas para poner a disposición del público productos oficiales del Club en las ciudades donde se disputen los partidos, así como una línea de merchandising específica de esta gira.

- Consolidación de la venta a través de Internet

FCBM ha llegado a un acuerdo con una empresa líder en el mercado europeo de venta on-line de material deportivo, para realizar la venta directa de los productos oficiales del Club a través de la nueva web blaugrana. Esta empresa ofrece como garantía su experiencia y liderazgo en el sector y una infraestructura segura y eficaz para soportar un negocio de ámbito global, que permitirá la venta del producto Barça por todo el mundo. 
- Remodelación de las instalaciones comerciales (La Botiga)

Nike aportará su amplia experiencia en este sector para consolidar el negocio de La Botiga. FCBM prevé un incremento de $15 \%$ de las ventas en La Botiga, objetivo que será posible a partir del mes de abril, cuando se completen las obras de remodelación de este punto de venta, ubicado en las mismas instalaciones del Camp Nou. Con una inversión de 600 mil pesetas, La Botiga se adaptará a las nuevas necesidades del mercado, con una imagen más actual y un nuevo concepto de distribución del espacio que mejore la disposición de los productos y la circulación interna de los clientes. El objetivo de este cambio es convertir La Botiga en el aparador ideal para los productos licenciados de la marca Barça.

\section{- Impacto económico}

Gracias al acuerdo entre el Club y Nike, que tiene vigencia hasta junio de 2008, FCB Merchandising explota la concesión de licencias y merchandising, los acuerdos internacionales de distribución comercial de la marca, la venta directa a través de Internet y la Botiga, con un compromiso similar a los que mantiene Nike con el Manchester United y la Juventus de Turín.

b) Los Angeles Lakers: la franquicia más cotizada de la $N B A^{21}$

- La empresa más productiva en las últimas temporadas, los Angeles Lakers,

21. Martínez, Hugo H. Los Angeles Lakers con la franquicia más cotizada de la NBA. Miami, 25 es la titular de la franquicia de más alto valor en la industria del baloncesto profesional de los Estados Unidos: 403 millones de dólares, según la más reciente valoración de la National Basquet Association (NBA), a marzo de 2002.

- La evaluación anual deja a los New York Knicks, 392 millones de dólares, como la segunda más cotizada de la Liga. En las siguientes calificaciones económicas figuran Chicago Bulls: 329 millones, y Portland Trailblazers: 283 millones.

- Elementos de la valoración:

- Rendimiento. Tres títulos consecutivos: 1999-2000, 2000-2001 y 2001-2002. Los Angeles Lakers son considerados como uno de los quintetos insignia de la NBA. De su nómina han salido grandes jugadores como Ervin «Magic» Jhonson y Kareem Abdul Jabbar.

- Su nuevo estadio, El Sttaples Center, le dio independencia y mayor presencia comercial.

- Merchandising. Ha aumentado en un $80 \%$, producto de los tres títulos conquistados en forma consecutiva.

- Calidad de sus activos corrientes (jugadores): tiene una nómina de lujo con deportistas de altísima cotización en el mercado de la NBA. Si se

de mar., 2002. Publicado en la revista electrónica DEPORTE\&NEGOCIOS ${ }^{\mathrm{TM}}$, Diario Económico y Financiero del Deporte. Autorizada su cita al autor de este trabajo por DEPORTE\&NEGOCIOS, (C) D\&N (contactenos@deporteynegocios.com). 
mira la sola línea titular de Los Lakers, se encuentran estrellas que tienen su propio respaldo deportivo. Shaquille O'Neal, ya ha ganado el título de mayor encestador de la Liga en temporada regular 1999-000 y fue el jugador más valioso de la post y temporada de 2000-01. Kobye Bryant, revelación del baloncesto norteamericano, para muchos el sucesor del gran Michael Jordan. Jugador explosivo y modelo comercial de la multinacional Adidas. Ron Harper, campeón en tres ocasiones con los Bulls de Chicago en la década de los 90 y uno de los mejores defensas en la NBA.

- Patrocinador. Por otro lado, el franquiciado de este superequipo, en cuanto a la distribución, comercialización y venta de ropa deportiva, es la multinacional Reebok. ¿A qué tiene derecho? A operar bajo una marca y utilizar ciertos sistemas de trabajo pertenecientes a Los Angeles Lakers. Tiene además, la facultad para trabajar en un territorio determinado. Goza del derecho de propiedad sobre su propia empresa; es decir, que Reebok puede, por ejemplo, establecer un contrato similar con los Indiana Pacers o cualquier otro equipo de la NBA

c) Yankees: la marca de mayor valor en la industria mundial del deporte 22

- Dos variables son las responsables de la calificación del valor de una empre-

22. Luckas E. Ascárraga. Yankees: la marca de mayor valor en la industria mundial del deporte. Nueva York, 13 de jul., 2002. Publicado en la revista electrónica DEPORTE\&NEGOCIOS ${ }^{\mathrm{TM}}$, sa deportiva en el mercado: marca y franquicia. Dos termómetros que miden el comportamiento productivo, principalmente en la exigente economía de consumo de los Estados Unidos, mercado que goza de las cuatro ligas más rentables del deporte mundial: MLB (béisbol), NFL (fútbol americano), NBA (baloncesto) y NHL (hockey sobre el hielo).

- Aunque la industria del deporte norteamericana goza de aceptación en muchos países con productos como el béisbol, el fútbol y el baloncesto, su rendimiento es más doméstico que internacional, pero de un alto valor económico a la hora de evaluar rendimientos.

- La marca Yankees de Nueva York, NY, una de las 30 filiales del holding de la Liga Profesional de Béisbol, MLB, es calificada por la consultora internacional FutureBrand como la más cotizada del mundo: 334 millones de dólares, tras evaluar el comportamiento económico, financiero y deportivo de las franquicias con fuerte presencia de consumo en el mercado norteamericano. Es de notar que esta consultora le da a los Yankees una valoración menor que la de la NBA.

- Para FutureBrand, el valor de una marca es resultado de la evaluación de los activos corrientes, rentabilidad financiera, flujos de caja, operaciones de mercadeo, infraestructura física (estadio) y rendimiento en los campos du-

Diario Económico y Financiero del Deporte. Autorizada su cita al autor de este trabajo por DEPORTE\&NEGOCIOS, (C) D\&N (contactenos@deporteynegocios.com). 
rante la más reciente temporada. El nombre del equipo de la NFL, Dallas Cowboys, 300 millones de dólares, es la segunda con más valor económico, luego de la de los Angeles Lakers, 272 millones.

- La franquicia. En enero de 2002, la revista Forbes publicó que la franquicia de los Yankees alcanzaba el tope en la MLB, 730 millones de dólares, valor apenas superado en el resto de la industria por la de los Washington Redskins, NFL, 796 millones, y la de los Dallas Cowboys, NFL, 743 millones.

- Una de las variables que incrementó el valor de las 30 franquicias de la MLB tras la temporada 2000-2001 fue el continuo ascenso de las nóminas, con salarios que el año pasado tocaron el techo de la industria deportiva norteamericana, generando temor por una posible recesión y, lo más grave, la huelga de «peloteros» si los empresarios no respetaban las tarifas acordadas.

- Otra variable que afecta tanto el valor de la franquicia como la cotización de la marca es el adecuado manejo de los derechos de televisión. En el caso de la MLB, los contratos con las cadenas Fox y NBC, ESPN y FOX Liberty Cable superan los 1587 millones de dólares.

\section{Reflexión final}

Como se ha podido apreciar, el concepto tradicional de relaciones públicas ha sufrido cambios a través del tiempo: se ha integrado o relacionado con el publicity, con el lobbying y otras formas de comu- nicación empresarial. El objetivo siempre ha sido generar una imagen favorable para la empresa. De otro lado, el área comercial de las empresas ha ido sofisticando cada vez más su manejo: el primer grupo de resultados han sido todos los instrumentos del marketing operativo (satisfactor, valor, distribución o ubicación y promoción); posteriormente se llegó a lo que se conoce como marketing estratégico, entendido como filosofía empresarial, consolidando la orientación total de la empresa al mercado. En el caso de las empresas deportivas, se ha aplicado además el merchandising, el patrocinio (sponsoring) y/ o el mecenazgo.

Sin embargo, los especialistas, tanto en relaciones públicas como en marketing, han descuidado en general el gran objetivo de todo negocio: maximizar la rentabilidad de la inversión. Los avances más notorios hacia ello son el valor de marca, el posicionamiento, las patentes, etc. Pero desde la óptica financiera siempre se ha trabajado con los conceptos de goodwill, know how y otros, como activos intangibles que se aprecian corrientemente en los estados financieros gerenciales $\mathrm{y}$, por ello mismo, tienen un valor patrimonial.

Modernamente se comienza a observar que las empresas, especialmente las deportivas, proyectan hacia sus públicos un complicado conjunto de comportamientos o acciones comunicacionales, cuyo resultado ha comenzado a denominarse imagen de empresa, y con la misma connotación que el concepto imagen de marca. Ello ha ido obligando a formalizar y profesionalizar el manejo de la imagen de la empresa deportiva, lo que ya ha hecho corriente el uso del término gerencia de imagen corporativa y -con el desarrollo de las ciencias de la comunica- 
ción-, la profesión de gerente de imagen corporativa.

Este trabajo ha buscado, precisamente, aportar al desarrollo de una mejor conceptualización y solidez de la gerencia de imagen corporativa aplicada a las empresas deportivas. Por ello, después del marco teórico el lector habrá podido apreciar que la gestión de grandes actividades deportivas, así como la de los clubes de las grandes ligas, incorpora cada vez con mayor evidencia los desarrollos modernos de gestión empresarial, fuertemente focalizados en el manejo de la imagen y con clara vocación por la aplicación de la teoría de la reputación; ello, a fin de lograr que el manejo financiero de los activos intangibles se materialice en una creciente y consistente valoración de la «marca» de la empresa deportiva o de la gran competencia deportiva internacional. 